\title{
PHASE-SEPARATION STRUCTURE OF POLYMER BLEND OF POLYSTYRENE AND POLYISOPRENE IN THE PRESENCE OF STYRENE-ISOPRENE DIBLOCK COPOLYMER
}

\author{
SHIGEKI TAKISHIMA, MIKIO KONNO, KUNIO ARAI \\ AND SHOZABURO SAITO \\ Department of Chemical Engineering, Tohoku University, Sendai 980
}

\begin{abstract}
Key Words: Thermodynamics, Phase Equilibrium, Micro Phase Separation, Polymer Alloy, Lamellar
\end{abstract} Structure, Polystyrene, Polyisoprene, Small Angle X Ray

\begin{abstract}
The micro-phase-separation structure of a system of homopolymers and a block copolymer was investigated theoretically and experimentally.

Phase-separation experiments were carried out for the blend system of homopolystyrene and homopolyisoprene in the presence of styrene-isoprene diblock copolymer, employing solvent casting as the method for the formation of phase separation. The experimental results revealed a lamellar structure in the micro-phase-separation, and domain spacings of the structure were measured.

A model for the lamellar structure of copolymer/homopolymer systems is proposed to predict the domain spacing on the basis of the statistical thermodynamic theory of Whitmore and Noolandi. The model can predict the domain spacing well in most cases.
\end{abstract}

\section{Introduction}

Polymer alloys, materials of multi-component polymer systems characterized by micro-phaseseparation structure, possess particular properties which do not appear in single-component materials. Since their properties are dependent on structural parameters such as the domain distance and shape of the micro-phase, prediction of the phase-separation structure is important for the design of polymer alloys.

Because of their great regularity, the phaseseparation structures of diblock copolymers have been the subject of extensive investigation. However, less attention has been given to those of homopolymer blends in the presence of block copolymers, in spite of their utilization potentialities.

In this work we have studied the phase-separation structure of a system composed of homopolymers and a block copolymer. The system of homopolystyrene and homopolyisoprene in the presence of styreneisoprene diblock copolymer was chosen because of the monodispersiveness of the molecular weight distribution and the clearness of the thermodynamic parameters of the components. The system is suitable for examining theoretical predictions of phaseseparation structure.

Recently, Whitmore and Noolandi ${ }^{11)}$ have reported a theoretical analysis of the phase-separation struc-

Received April 5, 1986. Correspondence concerning this article should be addressed to $S$. Saito. ture, applying statistical thermodynamics. They have proposed a predictive model for polymer systems which have spherical micelles as microdomains. In the present work, their theoretical analysis is extended to the lamellar structure of micro-phase separation. Predictions by the model are compared with the experimental results.

\section{Experimental}

\subsection{Materials}

The homopolystyrene (HS) was an anionically polymerized standard sample from Toyo Soda (code No. 5209). The homopolyisoprene (HI) and the styrene-isoprene diblock copolymer (C) were synthesized by living anionic polymerization according to Funabashi et al. $^{4)}$ In the polymerization, benzene was used as solvent, and sec-butyllithium as initiator. The polymerization conditions of the syntheses are shown in Table 1.

The properties of the polymers are presented in Table 2. Weight-average molecular weights, $\bar{M}_{w}$, and polydispersity index, $\bar{M}_{w} / \bar{M}_{n}$, were measured by gel permeation chromatography (Toyo Soda, HLC802UR) with tetrahydrofuran as solvent. Molecular weights of the homopolymers and each block of the copolymer were almost the same.

\subsection{Procedure}

Solvent casting was adopted as the method for the formation of the phase-separation structure. Benzene, which is a good solvent for each polymer component, was used as the casting solvent. Figure 1 shows the 
Table 1. Condition of anionic polymerization

\begin{tabular}{llcl}
\hline Initiator & $\begin{array}{c}\text { sec-Butyllithium/ } \\
n \text {-heptane soln. }\end{array}$ & $1.9 \times 10^{-4}$ & {$[\mathrm{~mol}]$} \\
& & \\
Solvent & Benzene & 260 & {$\left[\mathrm{~cm}^{3}\right]$} \\
Monomer 1 & Styrene & 10 & {$[\mathrm{~g}]$} \\
Monomer 2 & Isoprene & 10 & {$[\mathrm{~g}]$} \\
Temperature & & 308.2 & {$[\mathrm{~K}]$} \\
\hline
\end{tabular}

Table 2. Characterization of polymer samples used

\begin{tabular}{lll}
\hline Polymer & $\bar{M}_{w} \times 10^{-4}$ & $\bar{M}_{w} / \bar{M}_{n}$ \\
\hline C & $9.1(52.7 \mathrm{wt} \%$ polystyrene $)$ & 1.15 \\
HI & 4.3 & 1.25 \\
HS & 4.4 & 1.01 \\
\hline
\end{tabular}

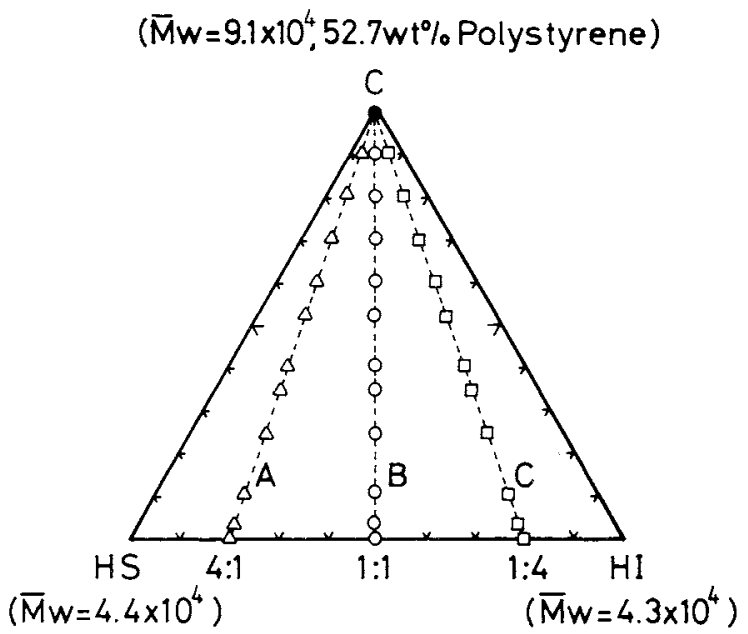

Fig. 1. Triangular diagram representing composition in weight fractions of ternary blend system composed of homopolystyrene $(H S)$, homopolyisoprene $(H I)$ and styreneisoprene diblock copolymer $(C)$.

compositions of the polymer mixtures used. The composition was varied along the lines of three different series (labeled A, B and C in Fig. 1) classified by the HS-to-HI weight ratios, $4: 1,1: 1$, and $1: 4$. Each polymer mixture was dissolved in benzene at $5 \mathrm{wt} \%$. The benzene solution was placed over mercury in a laboratory dish and kept for four days at $308.2 \mathrm{~K}$ to evaporate the solvent gradually. The polymer film formed on the mercury was dried under a vacuum for a few days until the weight of the film became constant.

The phase-separation structures of the polymer films were observed by transmission electron microscopy (TEM) using the osmium tetraoxide $\left(\mathrm{OsO}_{4}\right)$ fixation technique. Small-angle $\mathrm{X}$-ray scattering (SAXS) from the polymer films was also measured in order to investigate the average structure of the microdomains. The measured intensity distributions of X-ray scattering were corrected for absorption, air scattering and parasitic scattering according to usual methods.

\subsection{Experimental results}

TEM photographs of cast polymer films are presented in Fig. 2. In the photographs, the polyisoprene phase, which was stained with $\mathrm{OsO}_{4}$, is seen to be darker than the polystyrene phase. Photographs 2(a) to 2(e) belong to specimens of series B with the HS-toHI weight ratio of $1: 1$. The composition of the copolymer is written below each photograph in weight percent. The diblock copolymer specimen, containing no homopolymer, in photograph 2(a) reveals a high regularity of alternate lamellar structure in micro-phase separation. This structure is maintained in the range of copolymer content above $35 \mathrm{wt} \%$, while the regularity of the structure lessens with decreasing copolymer content. In the range below $35 \mathrm{wt} \%$, the lamellar structure appears only in a limited area and the copolymer is dispersed locally. With series $\mathrm{A}$ and $\mathrm{C}$, the lamellar structure is also observed at copolymer content above $30 \mathrm{wt} \%$, as seen in photographs $2(\mathrm{f})$ and $2(\mathrm{~g})$.

$X$-ray scattering patterns from specimens of series $B$ are plotted in Fig. 3. In the measurements, the Xray beam was irradiated parallel to the film surface. The scattered X-ray beam was detected along the direction normal to the film surface, because sharper scattering maxima were detected in this radiation direction than in the other radiation directions. For the specimens of copolymer content above $70 \mathrm{wt} \%$, the result shows a number of higher-order scattering maxima which indicate the existence of a long-range order in the spatial arrangements of the lamellar structure. With decreasing copolymer content the scattering maxima broaden or disappear and the number of scattering maxima is diminished, manifesting the disruption of the ordered structure. Similar results were obtained for the specimens of series $\mathrm{A}$ and $C$.

Using Bragg's equation, average domain spacings, $D$, were obtained from the scattering angles of the maxima. The experimental values of the average domain spacing could then be compared with predictions by our model.

\section{Prediction of the Phase-Separation Structure of Lamellar Morphology}

\subsection{Description of model}

Two predictive models for the phase-separation structure of the system of a diblock copolymer and a homopolymer have been proposed by Leibler et al., ${ }^{8)}$ and by Whitmore and Noolandi. ${ }^{11)}$ Both models are concerned with the structure of a spherical micelle of the copolymer dispersed in the matrix of the homopolymer. Although these models are very similar, the model of Whitmore and Noolandi seems to be more applicable, because they introduced a term representing the configurational entropy difference which 


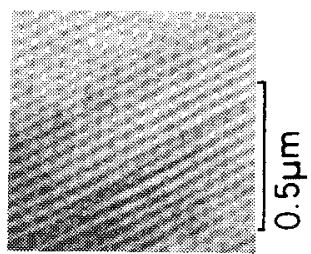

(a) $B-100$

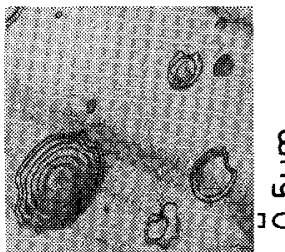

(e) $B-10$

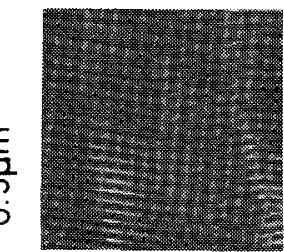

(b) $B-70$

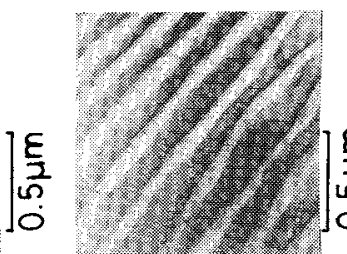

(c) $B-35$

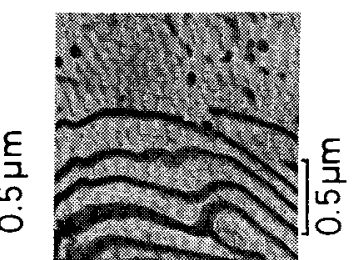

(d) $B-24$

Fig. 2. Transmission electron micrographs of ultrathin sections of cast polymer films stained with $\mathrm{OsO}_{4}$.

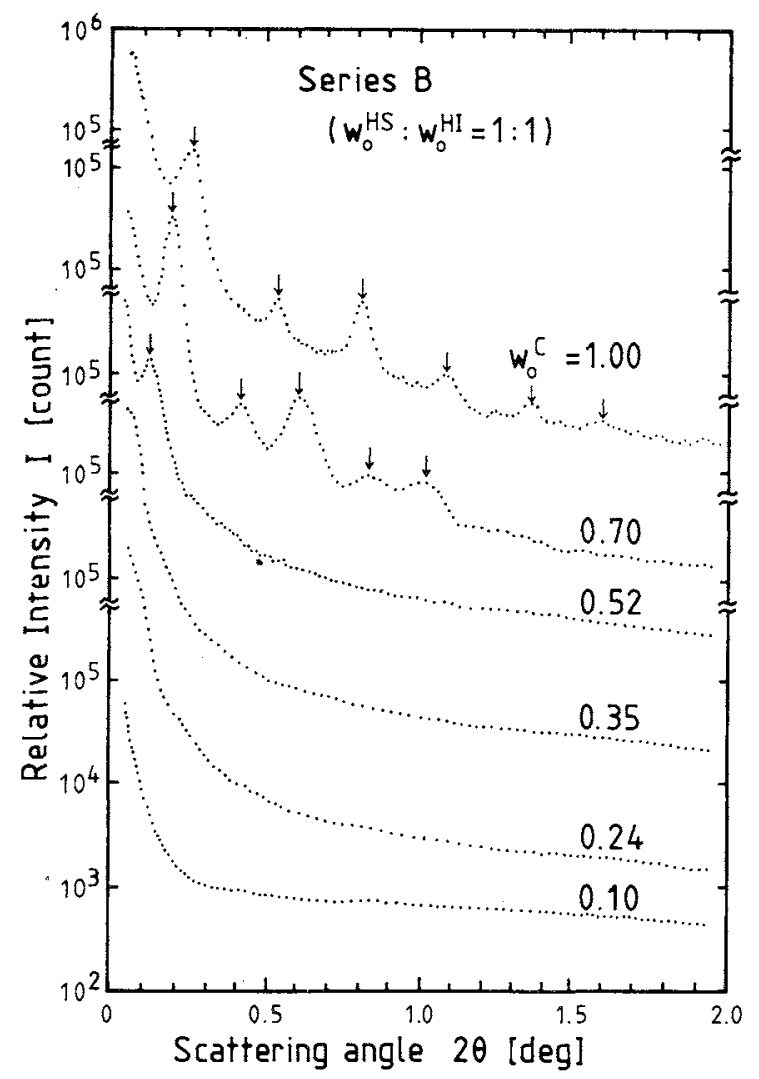

Fig. 3. SAXS intensity distributions from specimens of series $B$.

arises from the localization of joints of the copolymer. The present work adopted the model of Whitmore and Noolandi, assuming that their model for the isolated micelle system is applicable to prediction of the lamellar structure for copolymer/homopolymer systems with high regularity of arrangement.

Figure 4 shows the lamellar structure assumed in the present model. The structure consists of four different regions: [1], the region composed of HS only; [2], the region of HS and polystyrene block (CS) of

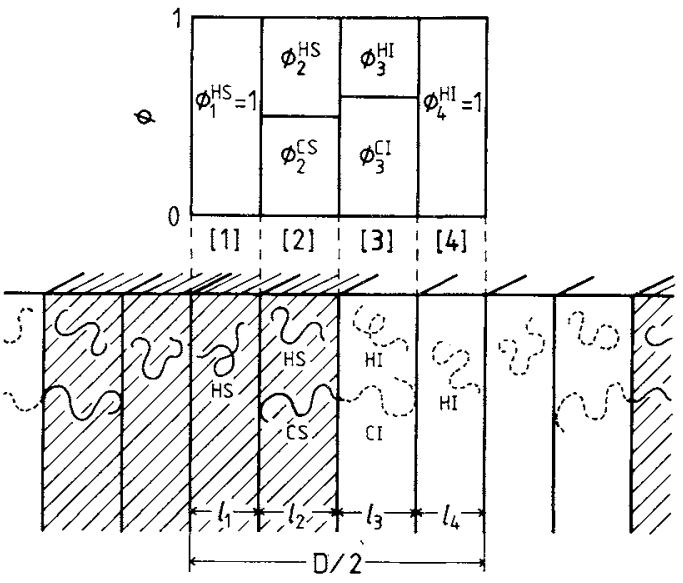

Fig. 4. Schematic shape of lamellar structure and volume fractions of each component.

copolymer; [3], the region of $\mathrm{HI}$ and polyisoprene block (CI) of copolymer; and [4], the region of HI only. Since polystyrene and polyisoprene are incompatible, HS and CS can be assumed not to penetrate into region [3] or [4], and vice versa. Using the thickness of region $i, l_{i}$, under the assumption that the system is incompressible, domain spacing $D$ and the fraction of total volume occupied by region $i, V_{i}$, are expressed as follows:

$$
\begin{gathered}
D=2\left(l_{1}+l_{2}+l_{3}+l_{4}\right) \\
V_{i}=2 l_{i} / D \quad(i=1,2,3,4)
\end{gathered}
$$

In terms of overall volume fraction of the copolymer $\phi_{0}^{C}\left(=\phi_{0}^{C S}+\phi_{0}^{C I}\right)$ and those of homopolymers $\phi_{0}^{H S}$ and $\phi_{0}^{H I}$, we have

$$
\begin{gathered}
\phi_{0}^{C S}=V_{2} \phi_{2}^{C S} \\
\phi_{0}^{C I}=V_{3} \phi_{3}^{C I} \\
\phi_{0}^{H S}=V_{1}+V_{2} \phi_{2}^{H S} \\
\phi_{0}^{H I}=V_{4}+V_{3} \phi_{3}^{H I}
\end{gathered}
$$


where

$$
\begin{aligned}
& \phi_{0}^{C S}=\phi_{0}^{C}\left(Z_{C S} / \rho_{O P S}\right) /\left(Z_{C} / \rho_{O C}\right) \\
& \phi_{0}^{C I}=\phi_{0}^{C}\left(Z_{C I} / \rho_{O P I}\right) /\left(Z_{C} / \rho_{O C}\right) \\
& Z_{C} / \rho_{O C}=Z_{C S} / \rho_{O P S}+Z_{C I} / \rho_{O P I}
\end{aligned}
$$

and $Z_{K}$ is the degree of polymerization of component $K, \rho_{O P S}$ and $\rho_{O P I}$ are the number densities of segments (a segment is regarded as a monomer unit in the treatment) of polystyrene and polyisoprene in pure state, and $\phi_{i}^{K}$ is the volume fraction of component $K$ in region $i$.

According to Whitmore and Noolandi, ${ }^{9,11}$ free energy difference per unit volume of the system relative to the random mixing state is presented as follows:

$$
\begin{aligned}
\Delta g & =\left(G_{\text {lamella }}-G_{\text {random }}\right) / \rho_{0} R T V \\
& =\Delta g_{\text {int }}+\Delta g_{H}+\Delta g_{J}+g_{e l}+g_{I}
\end{aligned}
$$

where $R$ is gas constant, $T$ is the absolute temperature, $V$ is the total volume of the system, and $\rho_{0}$ is a reference density. Each term on the right-hand side of Eq. (10) expresses the contributions of free energies explained below.

$\Delta g_{\text {int }}$ is a free energy difference arising from the interaction between polystyrene and polyisoprene in the bulk regions, so that this term is described by the interaction in the random mixing state:

$$
\Delta g_{\text {int }}=-\chi\left(\phi_{0}^{C S}+\phi_{0}^{H S}\right)\left(\phi_{0}^{C I}+\phi_{0}^{H I}\right)
$$

where $\chi$ is the interaction parameter of the FloryHuggins equation, ${ }^{2,7)}$ for a segment-pair of polystyrene and polyisoprene.

$\Delta g_{H}$ means a contribution from the configurational entropy of homopolymer molecules, which is caused by the restriction of regions where homopolymers can be placed:

$$
\begin{aligned}
\Delta g_{H}= & \frac{\rho_{O P S}}{\rho_{O}} \frac{\phi_{0}^{H S}}{Z_{H S}}\left(\phi_{1}^{H S} \ln \frac{\phi_{1}^{H S}}{V_{1}}+\phi_{2}^{H S} \ln \frac{\phi_{2}^{H S}}{V_{2}}\right) \\
& +\frac{\rho_{O P I}}{\rho_{0}} \frac{\phi_{0}^{H I}}{Z_{H I}}\left(\phi_{3}^{H I} \ln \frac{\phi_{3}^{H I}}{V_{3}}+\phi_{4}^{H I} \ln \frac{\phi_{4}^{H I}}{V_{4}}\right)
\end{aligned}
$$

The expressions for $\Delta g_{\text {int }}$ and $\Delta g_{H}$ can be calculated from the Flory-Huggins equation. ${ }^{2,7)}$

$g_{e l}$ represents the contribution of the elastic energy, arising from the stretching or compression of copolymer chains along the direction normal to the interface, and is given by Flory's expression:"

$$
g_{e l}=\frac{1}{2} \frac{\rho_{O C}}{\rho_{0}} \frac{\phi_{0}^{C}}{Z_{C}}\left(\alpha_{C S}^{2}+\alpha_{C I}^{2}+\frac{2}{\alpha_{C S}}+\frac{2}{\alpha_{C I}}-6\right)
$$

where

$$
\begin{aligned}
& \alpha_{C S}=\left(3 / Z_{C S}\right)^{1 / 2}\left(l_{2} / b_{P S}\right) \\
& \alpha_{C I}=\left(3 / Z_{C I}\right)^{1 / 2}\left(l_{3} / b_{P I}\right)
\end{aligned}
$$

and $b_{P S}$ and $b_{P I}$ are the Kuhn lengths for polystyrene and polyisoprene, respectively.

$\Delta g_{J}$ is a contribution from a decrease in the configurational entropy of the copolymer caused by the localization of joints, and can be expressed as the following equation: ${ }^{11)}$

$$
\Delta g_{J}=\frac{\rho_{0 C}}{\rho_{0}} \frac{\phi_{0}^{C}}{Z_{C}} \ln \frac{V_{J, \text { random }}}{V_{J, \text { lamella }}}
$$

where $V_{J, \text { random }}$ and $V_{J \text {, lamella }}$ are the volumes of regions where joints are present in the random mixing state and in the phase-separation state. The ratio of the volumes can be easily evaluated with the thickness of interface $t$, and Eq. (16) becomes

$$
\Delta g_{J}=\frac{\rho_{O C}}{\rho_{0}} \frac{\phi_{0}^{C}}{Z_{C}} \ln \frac{D}{2 t}
$$

The last term of the contributions to $\Delta g$ is the interfacial energy $g_{l}$, which is expressed as the product of interfacial tension $\gamma$ and interfacial area $A$. The interfacial area per unit volume $A$ equals $2 / D$, and then $g_{I}$ is expressed as

$$
g_{I}=\left(\gamma / \rho_{0} R T\right)(2 / D)
$$

The thickness of interface $t$ and the interfacial tension $\gamma$ can be estimated with Eqs. (18) and (19): ${ }^{11)}$

$$
\begin{gathered}
t=(2 / 3 \chi)^{1 / 2} b_{0} \\
\gamma / \rho_{0} R T=(\chi / 6)^{1 / 2} b_{0}
\end{gathered}
$$

where $b_{0}$ is a reference Kuhn length. The reference Kuhn length $b_{0}$ and the reference number density $\rho_{0}$ are given as

$$
\begin{aligned}
& b_{0}=\left(b_{P S}+b_{P I}\right) / 2 \\
& \rho_{0}=\left(\rho_{0 P S} \rho_{0 P I}\right)^{1 / 2}
\end{aligned}
$$

In the model of Whitmore and Noolandi, ${ }^{11)}$ an additional term for the contribution to the total free energy was included; the term corresponds to the transitional entropy of the spherical micelles. In the case of lamellar structure, this term determines the distributions of the thicknesses of homopolymer regions $l_{1}$ and $l_{4}$. However, it seems very difficult to estimate this entropy value. Moreover, the contribution of this term can be ignored under usual conditions. ${ }^{11)}$ Therefore, the effect of the transitional entropy is taken out of consideration and $l_{1}$ and $l_{4}$ are assumed to be constant in the present model.

In a case in which the overall volume fractions of each polymer $\phi_{0}^{C}, \phi_{0}^{H S}$, and $\phi_{0}^{H I}$ are given, three independent variables $l_{1}, l_{2}$, and $l_{3}$ can be taken. The total free energy $\Delta g$ can be minimized in respect of the range of these parameters. Values of the physical parameters used in the calculations are listed in Table 3. 
Table 3. Values of parameters used in the prediction

\begin{tabular}{cclc}
\hline Parameter & Value & Ref. \\
\hline$\chi$ & 0.127 & {$[-]$} & $10)$ \\
$b_{P S}$ & 0.68 & {$[\mathrm{~nm}]$} & $1)$ \\
$b_{P I}$ & 0.59 & {$[\mathrm{~nm}]$} & $6)$ \\
$\rho_{O P S}$ & $1.01 \times 10^{4}$ & {$\left[\mathrm{~mol} / \mathrm{m}^{3}\right]$} & $6)$ \\
$\rho_{O P I}$ & $1.36 \times 10^{4}$ & {$\left[\mathrm{~mol} / \mathrm{m}^{3}\right]$} & $6)$ \\
$z_{C S}$ & 462 & {$\left[1 / \mathrm{molecule}^{3}\right.$} & \\
$z_{C I}$ & 632 & {$[1 /$ molecule $]$} & \\
$z_{H S}$ & 423 & {$[1 /$ molecule $]$} & \\
$z_{H I}$ & 632 & {$[1 /$ molecule $]$} & \\
\hline
\end{tabular}

\subsection{Calculated results by the model}

Figure 5 shows the calculated results of $l_{i}$ and $D$ as a function of the total weight fraction of homopolymers $w_{0}^{H}\left(=w_{0}^{H S}+w_{0}^{H I}\right)$ for the polymer system of series A. In the range of small $w_{0}^{H}$, all homopolymers are solubilized into respective block domains composed of like components, and therefore the values of $l_{1}$ and $l_{4}$ are indicated as zero in the figure. The region of pure homopolystyrene [1] (defined in Fig. 4) appears at $w_{0}^{H}=0.05$, and that of pure homopolyisoprene [4] at $w_{0}^{H}=0.20$. The thicknesses of these homopolymer regions $l_{1}$ and $l_{4}$ as well as the domain spacing $D$ increase exponentially with increasing $w_{0}^{H}$. On the other hand, the thicknesses of copolymer regions $l_{2}$ and $l_{3}$ scarcely change over the whole range of composition.

The calculated results of $l_{i}$ and $D$ for the system of series B is shown in Fig. 6. This figure reveals a similar tendency to series $\mathrm{A}$, except that the $w_{0}^{H}$ values at which each region of pure homopolymers appears are close to each other. For the system of series C, the region of homopolyisoprene appears at a lower $w_{0}^{H}$ value than that of homopolystyrene.

For the polymer system of series $\mathrm{A}$, the volume fractions of homopolymers in regions [2] and [3], defined in Fig. 4, were calculated and are presented in Fig. 7. This figure also presents the number of joints per unit area of interface $N_{C} / A$, which is calculated by the following equation:

$$
\frac{N_{C}}{A}=\frac{l_{2}+l_{3}}{Z_{C S} /\left(\rho_{O P S} \phi_{0}^{C S}\right)+Z_{C I} /\left(\rho_{O P I} \phi_{0}^{C I}\right)}
$$

This value is considered to be important for study of the mechanical property of the interfacial region between unlike polymer phases. The calculated results of $\phi_{0}^{H S}$ and $\phi_{3}^{H I}$ show that the homopolymers can be dissolved in the block copolymer at most to the extent of about $10 \mathrm{vol} \%$. Consequently, addition of the homopolymers lowers the value of $N_{C} / A$ by $10 \%$ and no more. In such a case it might be expected that the interfacial region would have similar properties to those of the pure block copolymer.

Figure 8 presents the theoretically predicted and

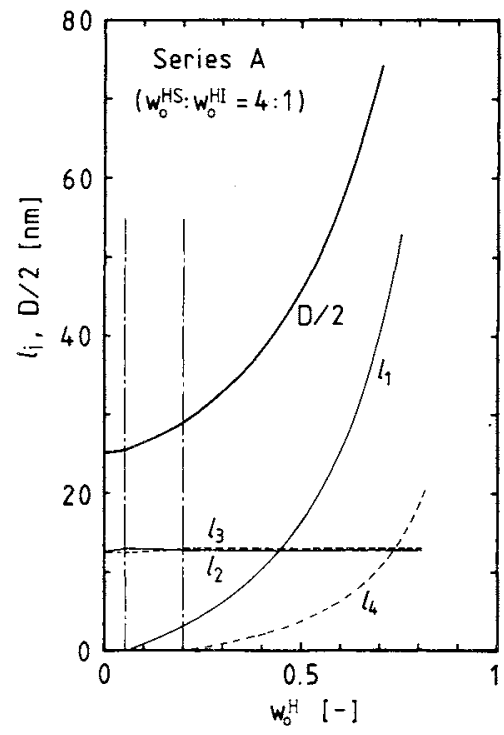

Fig. 5. Calculated results of thicknesses of each region $l_{i}$ and domain spacing $D$ as a function of total weight fraction of homopolymers $w_{0}^{H}$ for the system of series $\mathrm{A}$.

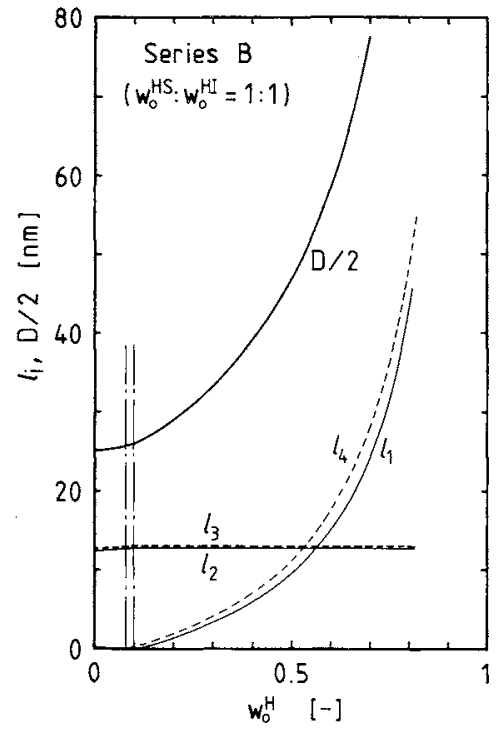

Fig. 6. Calculated results of $l_{i}$ and $D$ for the system of series B.

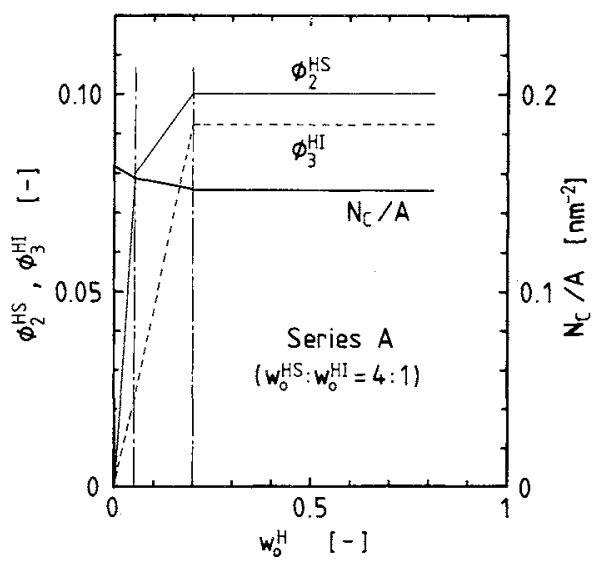

Fig. 7. Calculated results of volume fractions of homopolymers in regions [2] and [3], $\phi_{2}^{\mathrm{HS}}$ and $\phi_{3}^{H I}$, and number of joints per unit area of interface $N_{C} / A$ for the system of series $\mathrm{A}$. 


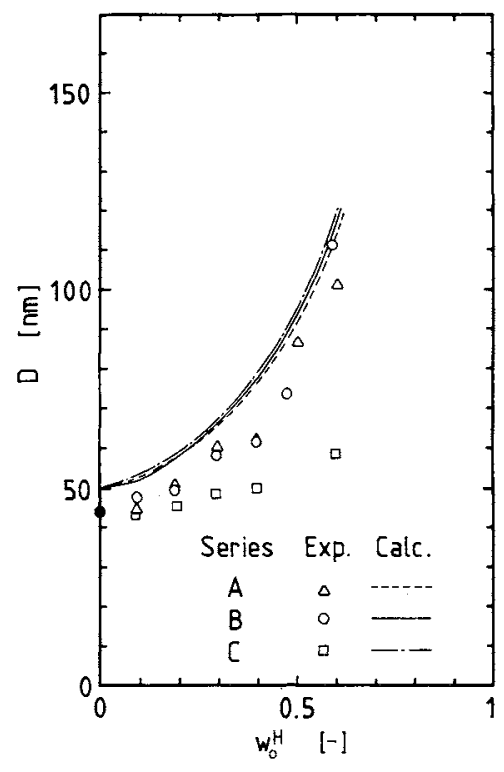

Fig. 8. Comparison between theoretically predicted and experimentally obtained results of domain spacing $D$.

experimentally obtained results of domain spacing $D$. Each set of experimental data for series A, B, and C shows a common tendency in which domain spacing $D$ increases with an increase in $w_{0}^{H}$. Qualitatively, this tendency is expressed by the model. For series A and $\mathrm{B}$, the model predicts values close to the experimental results of $D$. For series $C$, although there are no large discrepancies between the experimental and theoretical results in the range of relatively small $w_{0}^{H}$, the prediction is not yet satisfactory in the range of relatively large $w_{0}^{H}$.

Although there may be a number of reasons for these discrepancies, one may be the nonequilibrium effect, as was pointed out by Hashimoto et al. ${ }^{5)}$ This effect is encountered during solvent vaporization in film casting. Although benzene, the solvent used in this work, is known as a good solvent for both polystyrene and polyisoprene, the affinity of benzene is somewhat lower for polyisoprene than for polystyrene. In the polymer system swollen with the solvent, the polyisoprene phase contains the solvent at lower concentration than the other component, resulting in a reduction in diffusion rate of polyisoprene. Since the polymer system of series $\mathrm{C}$ contains the polyisoprene component at large volume fractions, especially in the range of low concentrations of the block copolymer, the reduction in the polyisoprene diffusion rate tends to make it difficult to attain an equilibrium state.

\section{Conclusion}

The phase-separation experiments involving the polymer system of homopolystyrene, homopolyisoprene and styrene-isoprene diblock copolymer showed that an alternate lamellar structure was formed over a wide range of composition. The average domain spacings of the lamella were measured by small-angle X-ray scattering.

The theoretical analysis of Whitmore and Noolandi was applied to derive a thermodynamic model for the structure of the lamellar arrangement. The domain spacings calculated by the model were compared with the experimental results. The model well predicted most of the experimental results, except for polymer systems containing polyisoprene at relatively large concentrations. It was suggested that these discrepancies might be attributed to a nonequilibrium effect.

\section{Acknowledgment}

This work was supported in part by a Grant-in-Aid for Scientific Research (No. 58430017) from the Ministry of Education, Science and Culture of Japan. and by the Asahi Glass Foundation for Industrial Technology, Tokyo, Japan.

The authors are indebted to Prof. T. Fujimoto of the Technological University of Nagaoka for his suggestions and instruction for the synthesis of polymers, and express sincere gratitude to Prof. N. Miyamoto of the Research Institute of Electrical Communication at Tohoku University for his valuable advice on small-angle $\mathrm{X}$-ray scattering analysis. Also, the authors would like to express their thanks to Dr. K. Itaya for his suggestions for the present work.

\section{Nomenclature}

$A \quad=$ area of interface $\left[\mathrm{m}^{2}\right]$

$b_{K} \quad=$ Kuhn length of component $K \quad$ [m]

$b_{0} \quad=$ reference Kuhn length [m]

$D \quad=$ domain spacing $\quad[\mathrm{m}]$

$G \quad=$ free energy $[\mathrm{J}]$

$g \quad=$ reduced free energy per unit volume $\quad[1 / \mathrm{mol}]$

$l_{i} \quad=$ thickness of region $i \quad[\mathrm{~m}]$

$\bar{M}_{n} \quad=$ number-average molecular weight $\quad[\mathrm{g} / \mathrm{mol}]$

$\bar{M}_{w} \quad=$ weight-average molecular weight $\quad[\mathrm{g} / \mathrm{mol}]$

$N_{C} \quad=$ number of joint [mol]

$\begin{array}{lll}R & =\text { gas constant } \quad[\mathrm{J} /(\mathrm{mol} \cdot \mathrm{K})]\end{array}$

$T \quad=$ absolute temperature $\quad[\mathrm{K}]$

$t \quad=$ thickness of interface $\quad[\mathrm{m}]$

$V \quad=$ total volume of system $\left[\mathrm{m}^{3}\right]$

$V_{i} \quad=$ fraction of total volume occupied by region $i\left[\mathrm{~m}^{3}\right]$

$w_{0}^{K} \quad=$ overall weight fraction of component $K \quad[-]$

$Z_{\mathrm{K}} \quad=$ degree of polymerization of component $K$

$[1 /$ molecule $]$

$\begin{array}{llr}\gamma & = & \text { interfacial tension } \\ \rho_{0} & =\text { reference number density of segments }\left[\mathrm{J} / \mathrm{m}^{2}\right] \\ \rho_{0 K} & {\left[\mathrm{~mol} / \mathrm{m}^{3}\right]} \\ & \text { number density of segments of polymer } K \\ \phi_{i}^{K} & \text { in pure state } & \text { volume fraction of component } K \text { in region } i \\ \phi_{0}^{K} & = & \text { overall volume fraction of component } K \\ \chi & = & \text { interaction parameter in the Flory-Huggins } \\ & \text { equation }\end{array}$

〈Superscripts and Subscripts〉

$C \quad=$ diblock copolymer

$C I \quad=$ polyisoprene block of copolymer

CS $\quad=$ polystyrene block of copolymer

HI = homopolyisoprene

HS = homopolystyrene

$i \quad=$ region 


$$
\begin{array}{ll}
P I & =\text { polyisoprene } \\
P S & =\text { polystyrene } \\
1,2,3,4 & =\text { region }
\end{array}
$$

\section{Literature Cited}

1) Ballard, D. G. H., G. D. Wignall and J. Schelten: Eur. Polym. $J ., 9,965$ (1973).

2) Flory, P. J.: J. Chem. Phys., 9, 660 (1941); 10, 51 (1942).

3) Flory, P. J.: "Principles of Polymer Chemistry," Cornell Univ Press, Ithaca, New York (1953).

4) Funabashi, H., Y. Miyamoto, Y. Isono, T. Fujimoto, Y. Matsushita and M. Nagasawa: Macromolecules, 16, 1 (1983).
5) Hashimoto, T., M. Fujimura and H. Kawai: Macromolecules, $13,1660(1980)$.

6) Hashimoto, T., N. Nakamura, M. Shibayama, A. Izumi and H. Kawai: Macromolecules Sci., Phys., B17, 389 (1980).

7) Huggins, M. L.: J. Phys. Chem., 9, 440 (1941).

8) Leibler, L., H. Orland and J. C. Wheeler: J. Chem. Phys., 79, 3550 (1983).

9) Noolandi, J. and K. M. Hong: Macromolecules, 16, 1443 (1983).

10) Rounds, N. A.: Doctoral Dissertation, Univ. Akron (1971).

11) Whitmore, M. D. and J. Noolandi: Macromolecules, 18, 657 (1985).

\title{
EFFECTS OF POST-IMPREGNATION DRYING CONDITIONS ON PHYSICAL PROPERTIES AND OVERALL REACTION RATE OF NICKEL/ALUMINA CATALYSTS
}

\author{
Yoshimitsu UEMURA, YASUO HATATE AND ATSUSHI IKARI \\ Department of Chemical Engineering, Kagoshima University, Kagoshima 890
}

Key Words: Chemical Reaction, Nickel Alumina Catalyst, Impregnation, Drying, Pore Structure, Intracatalyst Activity Profile, Effectiveness Factor, Benzene Hydrogenation

\begin{abstract}
The effects of post-impregnation drying conditions on the physical properties and overall rate of benzene hydrogenation were investigated with nickel/alumina catalysts, prepared by impregnating nickel ion-adsorptive and nickel ion-nonadsorptive aluminas and drying them under two conditions. Uniform nickel concentration profiles were obtained with the adsorptive support independent of the drying conditions, but the reduction degree of nickel by hydrogen and the nickel particle diameter were influenced by the drying conditions. With the nonadsorptive support, the drying conditions have a significant effect on the nickel profiles. In this case, the reduction degree and the particle diameter are dependent on the local all-nickel (reduced and unreduced nickel) content. The pore structure of the catalysts prepared from the nonadsorptive support was affected by the drying conditions. The experimental results of the overall reaction rate of benzene hydrogenation were found to be consistent with the change of the pore structure.
\end{abstract}

\section{Introduction}

Impregnation is one of the most important industrial methods for preparing supported catalysts. To obtain effective catalysts by impregnation, it is necessary to relate the physical properties and reactivity of the catalysts to the preparation conditions. Vincent $e t$ al. $^{23)}$ and Komiyama et $a l .{ }^{15)}$ quantitatively investigated the transport phenomena of active components within the pores of the support. Bartholomew et al. ${ }^{1)}$ reported the effects of calcination and reduction on the nickel morphology in nickel/alumina catalysts. Since a basic analytical study of the effectiveness factor of catalysts with

Received April 30, 1986. Correspondence concerning this article should be addressed to Y. Uemura. nonuniform activity profiles was accomplished by Kasaoka et al. ${ }^{13)}$ some analytical investigations ${ }^{2,3,12)}$ of more complex cases have been reported. Recently, some unique information and techniques, e.g., pore size control of alumina by the $\mathrm{pH}$ swing method, ${ }^{18)}$ measurement ${ }^{14)}$ of the radial profiles of nickel particle diameter in nickel/alumina catalysts, and the deformation $^{7)}$ of radial molybdenum profiles with molybdenum/alumina catalysts by high temperature calcination, were reported. However, few investigations $^{8)}$ involving the preparation, physical properties, and reactivity of impregnated catalysts are found. Especially, there is little information on the effects of post-impregnation drying conditions on the physical properties and reactivity of the catalysts.

In a previous study, ${ }^{22}$ we investigated nickel- 